\title{
Regulation of Aeroallergen Immunity by the Innate Immune System: Laboratory Evidence for a New Paradigm
}

\author{
Anthony A. Horner \\ Departments of Medicine and Pediatrics and The Sam and Rose Stein Institute for Aging, University of California, \\ San Diego, La Jolla, Calif., USA
}

\section{Key Words}

Allergy · Asthma • House dust extract • Hygiene hypothesis • Toll-like receptor $\cdot$ Lipopolysaccharide tolerance

\begin{abstract}
Over the last decade, it has become increasingly clear that innate responses to microbes are mediated largely by tolllike receptors (TLRs), which recognize a diverse family of molecules produced by viruses, bacteria and fungi. This article will present evidence that TLRs also play a dominant role in innate responses to non-infectious immunostimulatory materials present in house dust extracts (HDEs) and the living environments they represent. However, our investigations challenge the commonly held view that microbial products in ambient air protect against the allergic march by promoting protective Th1 biased responses to inspired aeroallergens. Instead, all HDEs studied to date have preferentially promoted the development of Th2 biased airway hypersensitivities when used as adjuvants for intranasal (i.n.) vaccination. In contrast, daily low dose i.n. HDE delivery was found to promote the development of aeroallergen tolerance. This article will review these experimental findings as evidence to propose a new paradigm by which airborne TLR ligands and other stimulants of innate immunity may influence aeroallergen specific immunity and the genesis of allergic respiratory diseases.

Copyright $\odot 2009$ S. Karger AG, Basel
\end{abstract}

\section{Introduction}

Asthma and other allergic diseases have become far more common in industrialized countries in recent decades, while atopy rates remain low in less developed countries [1-3]. Although reasons for these trends remain speculative, the rapidity with which allergic disease prevalence has increased in affected countries strongly suggests environmental factors are responsible. Adaptive responses associated with allergen tolerance and hypersensitivities appear to become imprinted early in life $[4,5]$. Therefore, as infants/toddlers spend a majority of their time indoors, there is a great deal of interest in determining how home exposures impact on allergic risk.

It is generally accepted that allergen exposure is a prerequisite for sensitization and for some allergens (i.e. cockroach and house dust mite), the risk for developing hypersensitivities increases significantly when levels in the home exceed a quantifiable threshold [6-8]. However, for other allergens (i.e. dogs, cats), increased levels of home exposure appear linked to a decreased risk of sensitization, both to the allergen of interest, and to unrelated allergens [8-10]. In a recent meta-analysis of 12 relevant studies, we found the odds ratio (OR) for development of allergic stigmata during childhood was 0.83 (CI $0.73-0.96$ ) in children raised with household pets versus those raised without them [11]. These and other findings

\section{KARGER}

(C) 2009 S. Karger AG, Basel

Fax +41613061234 E-Mail karger@karger.ch www.karger.com www.karger.com/jin
Dr. Anthony A. Horner

Departments of Medicine and Pediatrics and The Sam and Rose Stein Institute for Aging, University of California, San Diego

9500 Gilman Drive, Mail code 0663, La Jolla, CA 92093-0663 (USA)

Tel. +1 858534 5380, Fax +1 858534 5399, E-Mail ahorner@ucsd.edu 
suggest that aside from allergens themselves, living environments contain additional molecules that influence the immunological balance between allergen tolerance and hypersensitivity.

Endotoxin (TLR4), a molecule that activates immunocytes via TLR4, has been reported to be present at higher concentrations in homes with regular animal exposures than in homes with none $[12,13]$. Moreover, in several published reports, infants raised in high endotoxin homes were found to have a reduced incidence of atopic stigmata $[12,14]$. In our meta-analysis of 16 pertinent studies, the OR for allergen sensitization was 0.85 (CI 0.77-0.93) for infants raised in homes with high ambient endotoxin levels versus those raised in low levels[11]. In consideration of these relatively weak associations, it is important to recognize that endotoxin-rich environments generally contain increased levels of other immunostimulatory microbial products $[15,16]$. Furthermore, several manmade pollutants promote development of allergic hypersensitivities [17]. Although, much has been learned in recent years, the molecular complexity of ambient exposures has hampered efforts to develop a holistic understanding of their impact on atopic risk. Consistent with this theme, in additional meta-analyses, living on a farm (OR 0.74 , CI $0.61-0.91, \mathrm{n}=9$ ), living in close proximity with livestock (OR 0.54, CI 0.36-0.81, $\mathrm{n}=7$ ), and unpasteurized milk consumption during the first years of life (OR 0.67, CI $0.59-0.75, \mathrm{n}=5$ ) were all found to be far more negatively associated with allergic stigmata during childhood than were home endotoxin levels [11]. These meta-analyses and other epidemiological evidence strongly suggest that endotoxin levels are only one of a number of molecular variables influencing the allergenic potential of homes.

\section{Rationale for the Study of House Dust Extract Bioactivities}

While consensus opinion supports the view that living environments have a major educational influence on developing immune systems, understanding of how ambient exposures modify allergic risk remains highly speculative. By necessity, a majority of laboratory investigations aimed at characterizing how living environments affect host immunity have made a priori assumptions about which molecules are important. As an alternative, we reasoned that the immunological 'ether' associated with homes might be better understood by characterizing the immunostimulatory properties of clinically relevant but unpurified environmental samples. Logic sug- gests that gravity concentrates immunostimulatory particulates into settled dust. Moreover, house dust allergen and endotoxin concentrations have previously been found to be predictive surrogate markers of allergic risk. Therefore, we have begun to characterize the immunostimulatory properties of sterile house dust extracts (HDEs). Studies conducted to date have yielded provocative and reproducible results, which will be the focus of this paper [18-21].

\section{HDEs Activate Bone Marrow-Derived Dendritic Cells}

Dust samples were collected from the bedrooms of 15 suburban homes in San Diego (California, USA) and were processed by standardized techniques, which included suspension in PBS, physical agitation and sterile filtration [19, 21]. HDEs prepared in this manner were found to elicit bone marrow-derived dendritic cell (BMDDC) cytokine production in a concentration-dependent manner. Cytokines readily detected by ELISA included IL-6 and IL-12p40 [19]. However, HDE-induced BMDDC secretion of bioactive IL-12p70 was weak compared to responses induced by ligands for TLRs 4, 7 and 9 [21]. In unpublished experiments we recently observed that HDEs induce IL-23p19 mRNA synthesis, suggesting that HDEs may preferentially promote the synthesis of bioactive IL-23, a heterodimer of IL-12p40 and IL-23p19, rather than IL-12p70. Not surprisingly, HDEs were also found to induce the upregulation of MHC class II and a variety of co-stimulatory molecules on the surface of BMDDCs.

Consistent with other studies, mean endotoxin levels of dust samples obtained from homes with pets were more than twice those of samples obtained from homes without pets [19]. Moreover, a significant correlation was found between HDE endotoxin levels and their BMDDC cytokine inducing activity. However, the correlation coefficient for this association was less than 0.6, suggesting that additional molecules contribute to the bioactivities of HDEs.

To more specifically assess the contribution of TLRs to $\mathrm{HDE}$ responsiveness, HDE induced cytokine production by BMDDCs prepared from wild type (WT) and TLR and MyD88 knockout (ko) mice was compared [19]. In experiments with samples derived from multiple homes, TLR4 ko BMDDCs consistently demonstrated a marked reduction in $\operatorname{HDE}(\mathrm{n}=10)$ induced cytokine production and co-stimulatory molecule expression but residual responsiveness remained. TLR2 ko BMDDCs 
produced less IL- 6 than WT BMDDCs but IL-12p40 production and co-stimulatory molecule expression were relatively preserved. In contrast, HDE stimulated TLR9 ko BMDDCs produced less IL-6 and IL-12p40 than WT BMDDCs. Furthermore, while TLR4 ko BMDDCs displayed a greater deficit, HDE activated TLR9 ko BMDDCs expressed lower levels of co-stimulatory molecules than WT BMDDCs. These observations support the view that TLR4, TLR2 and TLR9 independently contribute to HDE mediated BMDDC responses. In a final series of experiments, WT and MyD88 ko BMDDC responses to HDE stimulation were compared [19]. HDE stimulated MyD88 ko BMDDCs were severely compromised in their ability to respond to HDEs, although residual cytokine production and co-stimulatory molecule upregulation could be detected. These results established that MyD88 dependent signaling pathways play a central role in mediating BMDDC responsiveness to HDEs.

\section{HDE Adjuvant Activities}

To assess the adjuvant activities of HDEs, mice were i.n. immunized with ovalbumin (OVA) alone or with HDE (prepared from 10 separate homes), 3 times, at weekly intervals [18]. A dose of $21 \mu \mathrm{l}$ of $\mathrm{HDE}(100 \mathrm{mg} / \mathrm{ml}$; concentration prior to filtration) was selected for these experiments, as preliminary experiments suggested that for most HDEs, this dose would provide optimal adjuvant activity. Additional control groups were i.n. immunized with OVA and Pam-3-Cys (TLR2), lipopolysaccharide (LPS; TLR4), or CpG DNA (TLR9), according to the same vaccination schedule. Mice i.n. immunized with OVA and HDE had far stronger adaptive responses, than mice i.n. immunized with OVA alone, establishing that HDEs have mucosal adjuvant activities. In addition, HDEs used in these studies were consistently found to act as Th2-biasing adjuvants, as they induced strong allergen-specific IgE and Th2 polarized cytokine responses but weak IgG2a and IFN $\gamma$ responses. Moreover mice immunized with OVA and HDEs consistently developed an eosinophil-rich airway inflammatory response and increased bronchial responsiveness to methacholine after i.n. OVA challenge [18]. Finally, most HDEs were found to be more effective at inducing OVA-specific, Th2-biased, airway hypersensitivities than Pam-3-Cys and lowdose LPS, both of which have previously been described as Th2 adjuvants [18, 22].

Consistent with previously discussed BMDDC studies, the adjuvant activities of HDEs were found to be de- pendent on signaling through MyD88 [18], further evidence of their relative dependence on signaling through TLRs. However, this evidence remains inconclusive, given that IL-1 and IL-18 signaling are also MyD88 dependent [23]. Therefore, this result could also reflect the importance of these cytokines in mediating the adjuvant activities of HDEs. Additional studies will be required to resolve this issue. Nonetheless, these experimental results challenge the commonly held belief that microbial products in general, and TLR ligands in particular, protect against the allergic march by inherently favoring the development of Th1-biased immune profiles. These findings might also lead one to wonder why mice and humans are not universally compromised by aeroallergen hypersensitivities.

\section{HDE Tolerogenic Activities}

The experiments just discussed might be construed to suggest that many, if not all, living environments intrinsically promote the development of allergic asthma. However, in these studies mice were airway exposed to the immunostimulatory contents of HDEs at weekly intervals and at levels likely to be in great excess of daily physiological exposures. In contrast, individuals are thought to inhale air laced with far lower concentrations of immunostimulatory molecules on a semi-continuous basis [24]. Therefore, additional experiments were designed to better model real-world exposures. First a HDE standard (HDEst) was prepared from individual HDEs with high levels of adjuvant activity. Mice received 3 weekly i.n. OVA immunizations, as in previously described experiments. HDEst was delivered i.n. weekly with OVA, or daily in low doses (1/7th weekly dose, $3 \mu$ l; beginning 1 week before the first and ending with the last dose of OVA, as in previously discussed experiments), or both daily and weekly [18].

Daily i.n. HDEst delivery had little adjuvant effect on OVA-specific responses. More importantly, this delivery schedule prevented mice concurrently receiving weekly i.n. OVA and HDEst (adjuvant dose) from developing both Th2-biased adaptive responses and experimental asthma [18]. In additional unpublished studies we determined whether weekly i.n. OVA vaccination in conjunction with daily HDEst delivery would induce long lasting allergen specific tolerance. In these studies mice received a series of 3 weekly i.n. OVA vaccinations either alone, with weekly adjuvant doses of HDE $(21 \mu \mathrm{l})$, or in conjunction with daily low-dose HDEst $(3 \mu \mathrm{l})$ delivery, as 
just described. One month after the last of the primary OVA immunizations, all mice were OVA sensitized by weekly i.n. OVA/adjuvant dose HDEst delivery on 3 occasions. Mice receiving i.n. OVA and daily HDEst during primary immunizations were found to be highly resistant to Th2 sensitization, while mice in other primary immunization groups (nothing, OVA alone or weekly OVA plus HDEst) were not. In additional studies, we established that both the Th2 adjuvant and tolerogenic activities of HDEst could be replicated with purified LPS. To our knowledge, these are the first experiments to directly establish that stimulants of innate immunity, which are traditionally considered to be adjuvants, when used under distinct experimental conditions, can also serve as potent inducers of antigen tolerance.

\section{Modulatory Influence of HDE on the Th2-Biased Airway Hypersensitivity Response}

Along with infants developing primary allergen-specific immunity (hypersensitivity vs. tolerance), airway exposures to stimulants of innate immunity are likely to impact greatly on the clinical status of patients with preexisting allergic respiratory diseases. Therefore, we determined how bolus and daily i.n. delivery of HDEst modified the aeroallergen challenge responses of previously Th2-sensitized mice [20]. One group of mice received a high-dose HDEst bolus concurrently with each i.n. OVA challenge. Another group of mice received low dose i.n. HDEst (1/7th bolus dose) on a daily basis beginning 7 days before the first and ending with the final OVA challenge. Somewhat surprisingly, both daily and bolus i.n. HDEst delivery attenuated features of the airway hypersensitivity response, but daily delivery was significantly more effective. Moreover, daily but not bolus HDEst delivery attenuated bronchial lymph node mononuclear cell cytokine responses to in vitro OVA stimulation and partially desensitized mice to additional airway challenges a month later with OVA alone.

Similar to daily i.n. HDEst delivery, daily i.n. LPS delivery was found to attenuate the airway hypersensitivity response of Th2 sensitized mice undergoing airway allergen challenge [20]. However, unlike many other comparative studies in which HDE and LPS were found to have similar bioactivities, in Th2 sensitized mice undergoing airway allergen challenge, concurrent bolus delivery of HDEst and LPS had divergent effects. As previously mentioned, bolus HDEst delivery attenuated the airway hypersensitivity response, while with LPS, the airway inflammatory response was augmented [20]. In a final series of experiments, daily i.n. HDEst delivery was found to efficiently attenuate the airway hypersensitivity response of previously Th2-sensitized TLR4-deficient mice undergoing concurrent allergen challenge. Taken together, these observations demonstrate that endotoxin does not completely recapitulate, nor is it completely responsible for the immunomodulatory potential of living environments.

\section{Conclusions}

Investigations reviewed in this paper demonstrate that pulmonary exposures to allergen non-specific immunostimulants ubiquitous in indoor living environments have the potential to impact significantly on allergic risk. Nonetheless, understanding of the molecular variables and immunological mechanisms responsible is far from complete. Correlations between pet, farm, animal, unpasteurized milk and endotoxin exposures during childhood and a reduced incidence of allergic manifestations have been found in some studies but these trends have been inconsistently reported, and in select studies associations were relatively weak, nonexistent or reversed [11]. Moreover, results from the many epidemiological studies conducted to date provide little insight into the cellular and molecular mechanisms by which living environments influence allergic risk.

Although ubiquitous, concentrations of TLR ligands and other stimulants of innate immunity in the ambient air of most micro-environments are thought to be very low. For example, one study of pediatric exposure calculated that the median amount of endotoxin inhaled by children each day is from 46 to $220 \mathrm{pg}$ [24]. However, endotoxin can be readily detected in essentially all air samples tested and concentrations can vary by as much as 5 logs $[24,25]$. Given this lack of homogeneity, estimates of daily exposure based on air sampling are unlikely to account for episodic periods of exposure to air containing extremely high concentrations of endotoxin and/or other immunostimulants of microbial origin. Moreover, despite evidence that living environments contain a wide range of molecules that induce innate immune activation, the only assay commercially available for their detection at a molecular level is the limulus lysate assay for endotoxin. This deficiency in assays for the detection of molecules with immunostimulatory potential is a major impediment for efforts aimed at characterizing how living environments affect allergic risk. 
As an alternative to measuring concentrations of immunostimulatory molecules in HDEs our research group has used well-defined bioassays and animal models of allergic disease to better characterize the bioactivities of HDEs and the living environments they represent. In vitro studies have shown that TLRs play a central role in dendritic cell responsiveness to the allergen non-specific immunostimulatory contents of unpurified HDEs and the living environments they represent $[19,21]$. Additional i.n. vaccination experiments have revealed that weekly airway exposures to adjuvant doses of HDEs induce Th2biased airway hypersensitivities to co-administered allergen, while daily HDE exposures promote the development of long-lived allergen tolerance [18]. In Th2-sensitized mice undergoing airway challenge, both daily and bolus HDE delivery were found to attenuate the Th2-biased airway hypersensitivity response, but again only daily HDE delivery promoted long-lived aeroallergen desensitization [20].

If reflective of real world exposures, our HDE studies strongly suggest that TLR ligands and other allergen nonspecific immunostimulatory molecules that become aerosolized and inhaled do not inherently drive the development of 'protective' Th1-biased responses to aeroallergens, as commonly suggested in the literature. Instead, these findings suggest that living environments have far greater potential to promote tolerogenic and/or Th2-biased immune responses, depending on additional variables $[18,20]$. These are likely to include the relative and absolute concentration of each individual immunostimulant contained in inspired air during the course of the day, and in particular, the frequency and duration of exposures to air laced with high concentrations of these immunostimulants. The observation that molecules that activate dendritic cells and act as mucosal adjuvants (i.e. HDEs and LPS) also function as tolerogenic agents is novel and likely to be of great clinical significance. However, while mechanistic studies are ongoing, current understanding of this phenomenon is limited and based principally on indirect evidence.

Along with attenuating the adjuvant activities of HDEs, we found that the innate airway inflammatory response that develops $24 \mathrm{~h}$ after i.n. bolus HDE challenge (neutrophilic inflammation and cytokine release) can be inhibited by pre-treating mice with daily i.n. low dose HDE [18]. This phenomenon of reduced responsiveness with repetitive exposure has previously been described with LPS and other TLR ligands, and is commonly referred to as LPS tolerance [26-28]. In unpublished studies we further observed that daily i.n. HDE delivery led to significant increases in local mRNA expression for molecules that mediate LPS tolerance(IL-10, STAT3, IRAKM, SHIP) $[26,28-30]$ when compared to bolus i.n. HDE delivery. These observations may explain why human lungs remain un-inflamed despite the continuous inhalation of air laced with stimulants of innate immunity [31]. These preliminary findings also provide evidence that mechanisms associated with the regulation of innate immunity (LPS tolerance) might contribute to the tolerogenic influence of daily i.n. HDE/LPS delivery on allergen specific immunity.

Over the last several years, our laboratory has characterized the immunstimulatory potential of homes, using HDEs as a surrogate material for investigations presented herein [18-21]. These studies lead us to conclude that TLRs play a central role in mediating responses to immunostimulants that are ubiquitous indoors. However, in urban environments, diesel exhaust and other pollutants of human origin are likely to be found at far higher concentrations out of doors and these molecules are known to perturb immune homeostasis, at least in part, by creating oxidant stress, a phenomenon that is uniquely receptor independent [17]. Several laboratories have begun to investigate the immunostimulatory potential of unpurified samples of particulate matter (APM) prepared from outdoor air [32-35]. Moreover, Williams et al. [32] have already published that APM has MyD88 independent immunostimulatory properties. Given these considerations, it will be important to compare the immunostimulatory properties of APM and diesel exhaust particulates with those of HDEs in future experiments.

While much remains to be learned of the molecular and immunostimulatory content of inhaled air, experimental results discussed in this article lead us to propose a new paradigm by which ambient exposures might modulate innate immune homeostasis in the airways, promote the development of aeroallergen tolerance or hypersensitivity, and modify the respiratory status of previously aeroallergen-sensitized patients. The tenets of this model (fig. 1) are as follows. (1) Basal levels of exposure to endotoxin and other immunostimulatory materials present in ambient air are generally not sufficient to provoke an inflammatory reaction in the airways or to provide adjuvant activity for co-inspired aeroallergens. (2) Physiological exposures to ambient air laced with low but adequate concentrations of TLR ligands and other immunostimulatory molecules leads to a state of relative airway hypo-responsiveness to these molecules and potentially other immunostimulants. (3) Airways receiving inadequate innate immune stimulation remain far more 


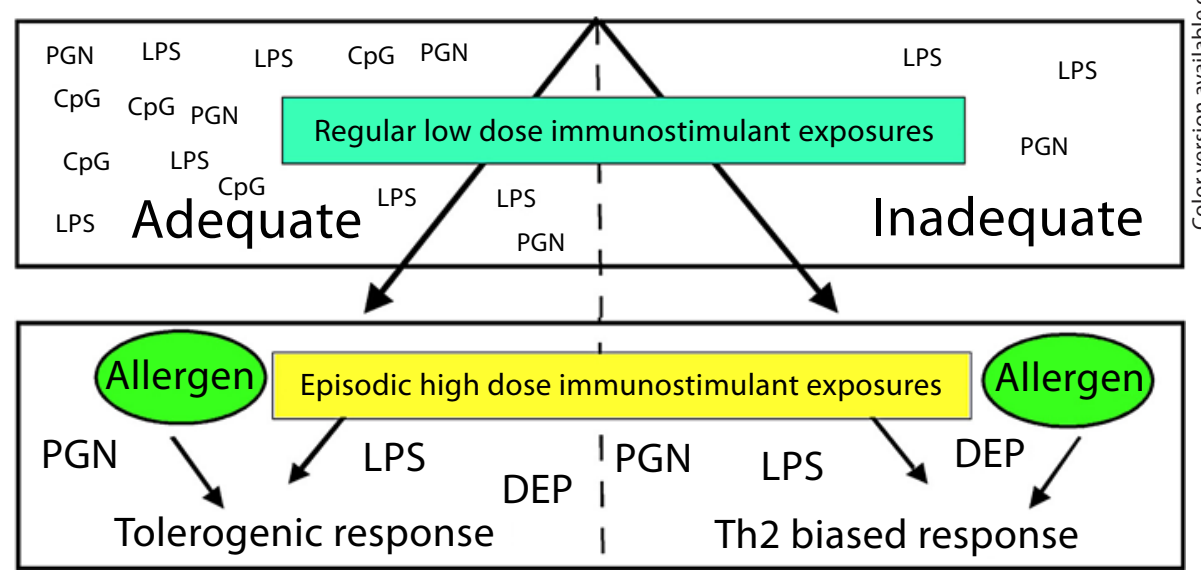

Fig. 1. Ambient allergen non-specific immunostimulants and the regulation of aeroallergen specific immunity. This diagram presents a paradigm by which adaptive responses to aeroallergens might be regulated by ambient exposures to molecules that activate the innate immune system. Evidence in support of this model is reviewed in the body of this article. The paradigm proposes that if an individual's airways are exposed to adequate concentrations of TLR ligands and potentially other immunostimulants on a regular basis, then he or she will become functionally desensitized to their Th2 adjuvant potential and aeroallergen tolerance will be promoted. However, if basal levels of airway immune stim-

responsive to airborne immunostimulants than airways that are regularly exposed to these molecules. (4) If dampening by basal immunostimulant exposures is inadequate, episodic exposures to ambient air laced with super-physiologic concentrations of these molecules have the potential to provide Th2 adjuvant activity for the development of hypersensitivities to co-inspired aeroallergens.

Although far from proven, the model presented in figure 1 is testable and consistent with current understanding of the regulation of innate and adaptive immunity in the airways. In addition, this paradigm provides a rational mechanistic framework for understanding why semicontinuous airway exposures to aeroallergens and molecules that activate innate immunity only prime a subset of individuals to develop Th2-biased allergic respiratory diseases, while a majority become aeroallergen tolerant and their airways remain not inflamed. In the years to come, the continued study of the interface between host immunity and the environment will give rise to a far more comprehensive understanding of the genesis of allergic respiratory diseases and other diseases of immune ulant exposure are inadequate, then during infrequent periods of exposure to ambient air containing aeroallergens and high concentrations of immunostimulants, individuals remain vulnerable to the development of allergen hypersensitivities. While incomplete, the figure includes select immunostimulants, identified by acronyms, that can be readily detected in ambient air and/or other environmental samples. LPS = Lipopolysacchride from Gramnegative bacteria; PGN = peptidoglycan from bacterial cell walls; $\mathrm{CpG}=\mathrm{CpG}$ containing microbial DNA; DEP = diesel exhaust particulate.

dysregulation and ultimately, may lead to the development of better therapeutic strategies for their prevention and treatment.

\section{Acknowledgments}

This work was supported by grant AI61772 from the National Institutes of Health.

References

$\checkmark 1$ Horner AA: Toll-like receptor ligands and atopy: a coin with at least two sides. J Allergy Clin Immunol 2006;117:1133-1140.

2 Braman SS: The global burden of asthma. Chest 2006;130:4S-12S

- 3 Keller MB, Lowenstein SR: Epidemiology of asthma. Semin Respir Crit Care Med 2002; 23:317-329.

-4 Prescott SL, Macaubas C, Smallacombe T, Holt BJ, Sly PD, Holt PG: Development of allergen-specific T-cell memory in atopic and normal children. Lancet 1999;353:196-200. 
5 Prescott SL, Macaubas C, Holt BJ, Smallacombe TB, Loh R, Sly PD, Holt PG: Transplacental priming of the human immune system to environmental allergens: universal skewing of initial $\mathrm{T}$ cell responses toward the Th2 cytokine profile. J Immunol 1998;160: 4730-4737.

6 Platts-Mills TA, Ward GW Jr, Sporik R, Gelber LE, Chapman MD, Heymann PW: Epidemiology of the relationship between exposure to indoor allergens and asthma. Int Arch Allergy Appl Immunol 1991;94:339345.

7 Huss K, Adkinson NF Jr, Eggleston PA, Dawson C, Van Natta ML, Hamilton RG: House dust mite and cockroach exposure are strong risk factors for positive allergy skin test responses in the Childhood Asthma Management Program. J Allergy Clin Immunol 2001;107:48-54.

$\checkmark 8$ Platts-Mills TA, Woodfolk JA, Erwin EA, Aalberse R: Mechanisms of tolerance to inhalant allergens: the relevance of a modified Th2 response to allergens from domestic animals. Springer Semin Immunopathol 2004; 25:271-279.

$\checkmark 9$ Frew AJ: Advances in environmental and occupational diseases 2004. J Allergy Clin Immunol 2005;115:1197-1202.

10 Ownby DR, Johnson CC: Does exposure to dogs and cats in the first year of life influence the development of allergic sensitization? Curr Opin Allergy Clin Immunol 2003;3: 517-522.

-11 Tse K, Horner AA: Defining a role for ambient TLR ligand exposures in the genesis and prevention of allergic diseases. Semin Immunopathol 2008;30:53-62.

-12 Braun-Fahrlander C, Riedler J, Herz U, Eder W, Waser M, Grize L, Maisch S, Carr D, Gerlach F, Bufe A, Lauener RP, Schierl R, Renz $H$, Nowak D, von Mutius E: Environmental exposure to endotoxin and its relation to asthma in school-age children. N Engl J Med 2002;347:869-877.

-13 Gereda JE, Klinnert MD, Price MR, Leung DY, Liu AH: Metropolitan home living conditions associated with indoor endotoxin levels. J Allergy Clin Immunol 2001;107: 790-796.

$\checkmark 14$ Gereda JE, Leung DY, Thatayatikom A, Streib JE, Price MR, Klinnert MD, Liu AH: Relation between house-dust endotoxin exposure, type $1 \mathrm{~T}$-cell development, and allergen sensitisation in infants at high risk of asthma. Lancet 2000;355:1680-1683.
15 Roy SR, Schiltz AM, Marotta A, Shen Y, Liu AH: Bacterial DNA in house and farm barn dust. J Allergy Clin Immunol 2003;112:571578.

16 van Strien RT, Engel R, Holst O, Bufe A, Eder W, Waser M, Braun-Fahrlander C, Riedler J, Nowak D, von Mutius E: Microbial exposure of rural school children, as assessed by levels of N-acetyl-muramic acid in mattress dust, and its association with respiratory health. J Allergy Clin Immunol 2004;113:860-867.

17 Saxon A, Diaz-Sanchez D: Air pollution and allergy: you are what you breathe. Nat Immunol 2005;6:223-226.

18 Ng N, Lam D, Paulus P, Batzer G, Horner AA: House dust extracts have both Th2 adjuvant and tolerogenic activities. J Allergy Clin Immunol 2006;117:1074-1081.

19 Boasen J, Chisholm D, Lebet L, Akira S, Horner AA: House dust extracts elicit Tolllike receptor-dependent dendritic cell responses. J Allergy Clin Immunol 2005; 116 : 185-191.

20 Lam D, Ng N, Lee S, Batzer G, Horner AA: Airway house dust extract exposures modify allergen-induced airway hypersensitivity responses by TLR4-dependent and independent pathways. J Immunol 2008;181:29252932.

21 Batzer G, Lam DP, Paulus P, Boasen J, Ng N Horner AA: Using house dust extracts to understand the immunostimulatory activities of living environments. Immunobiology 2007;212:491-498.

$\checkmark 22$ Eisenbarth SC, Piggott DA, Huleatt JW, Visintin I, Herrick CA, Bottomly K: Lipopolysaccharide-enhanced, toll-like receptor 4 -dependent $\mathrm{T}$ helper cell type 2 responses to inhaled antigen. J Exp Med 2002;196:16451651.

23 Adachi O, Kawai T, Takeda K, Matsumoto M, Tsutsui H, Sakagami M, Nakanishi K, Akira S: Targeted disruption of the MyD88 gene results in loss of IL-1- and IL-18-mediated function. Immunity 1998;9:143-150.

24 Rabinovitch N, Liu AH, Zhang L, Rodes CE, Foarde K, Dutton SJ, Murphy JR, Gelfand EW: Importance of the personal endotoxin cloud in school-age children with asthma. J Allergy Clin Immunol 2005; 116:1053-1057.

25 Platts-Mills JA, Custis NJ, Woodfolk JA, Platts-Mills TA: Airborne endotoxin in homes with domestic animals: implications for cat-specific tolerance. J Allergy Clin Immunol 2005;116:384-389.
26 Sly LM, Rauh MJ, Kalesnikoff J, Song CH, Krystal G: LPS-induced upregulation of SHIP is essential for endotoxin tolerance. Immunity 2004;21:227-239.

27 Jacinto R, Hartung T, McCall C, Li L: Lipopolysaccharide- and lipoteichoic acid-induced tolerance and cross-tolerance: distinct alterations in IL-1 receptor-associated kinase. J Immunol 2002;168:6136-6141.

-28 Fan H, Cook JA: Molecular mechanisms of endotoxin tolerance. J Endotoxin Res 2004; 10:71-84.

29 Kobayashi K, Hernandez LD, Galan JE, Janeway CA Jr, Medzhitov R, Flavell RA: IRAK$\mathrm{M}$ is a negative regulator of Toll-like receptor signaling. Cell 2002;110:191-202.

$>30$ Escoll P, del Fresno C, Garcia L, Valles G, Lendinez MJ, Arnalich F, Lopez-Collazo E: Rapid up-regulation of IRAK-M expression following a second endotoxin challenge in human monocytes and in monocytes isolated from septic patients. Biochem Biophys Res Commun 2003;311:465-472.

$>31$ Alexis NE, Eldridge MW, Peden DB: Effect of inhaled endotoxin on airway and circulating inflammatory cell phagocytosis and CD11b expression in atopic asthmatic subjects. J Allergy Clin Immunol 2003;112:353361 .

>32 Williams MA, Porter M, Horton M, Guo J, Roman J, Williams D, Breysse P, Georas SN: Ambient particulate matter directs nonclassic dendritic cell activation and a mixed TH1/TH2-like cytokine response by naive CD4+ T cells. J Allergy Clin Immunol 2007; 119:488-497.

33 Zhou H, Kobzik L: Effect of concentrated ambient particles on macrophage phagocytosis and killing of Streptococcus pneumoniae. Am J Respir Cell Mol Biol 2007;36:460465.

34 Alexis NE, Lay JC, Zeman K, Bennett WE, Peden DB, Soukup JM, Devlin RB, Becker S: Biological material on inhaled coarse fraction particulate matter activates airway phagocytes in vivo in healthy volunteers. J Allergy Clin Immunol 2006;117:1396-1403.

35 Williams MA, Rangasamy T, Bauer SM, Killedar S, Karp M, Kensler TW, Yamamoto M, Breysse P, Biswal S, Georas SN: Disruption of the transcription factor Nrf2 promotes pro-oxidative dendritic cells that stimulate Th2-like immunoresponsiveness upon activation by ambient particulate matter. J Immunol 2008;181:4545-4559. 